This document is the unedited Author's version of a Submitted Work that was subsequently accepted for publication in ACS Applied Materials and Interfaces, copyright (c) American Chemical Society after peer review. To access the final edited and published work see:

https://dx.doi.org/10.1021/acsami. 8b22335. 


\title{
Solid materials with tunable reverse photochromism
}

\author{
Alex Julià López, ${ }^{\#}$ Daniel Ruiz-Molina, ${ }^{\#}$ Jordi Hernando, ${ }^{* \ddagger}$ Claudio Roscini. ${ }^{*, \#}$ \\ \# Catalan Institute of Nanoscience and Nanotechnology (ICN2), CSIC and The Barcelona Institute of Science and Technology \\ (BIST), Campus UAB, Bellaterra, Barcelona o8193, Spain
}

‡Universitat Autònoma de Barcelona, Edifici C/n, Campus UAB, Cerdanyola del Vallès, o8193, Spain

reverse/negative photochromism, spiropyrans, merocyanine forms, microcapsules, rewritable devices

\begin{abstract}
Herein we report on a novel, straightforward and universal strategy to achieve solid materials with highly tunable reverse photochromism. This was accomplished by means of commercially available spiropyran dyes, which can produce different types of stable merocyanine states (i.e. non-protonated and protonated forms) displaying distinct reverse photochromic properties (i.e. colours and colouration rates). To finely control the concentration ratio of these species and, as such, tailor the optical performance of the photochromes, we exploited their differential interaction with surrounding media of distinctive nature (i.e. non-volatile protic and aprotic polar solvents). In this way, solutions displaying different photochromic responses were prepared for individual spiropyrans without requiring chemical derivatization, an approach that can be generalized to other spiro dyes with distinct acid-base properties. To transfer this behaviour to the solid state, core-shell capsules of these solutions were prepared, which were then used as ink materials for the fabrication of flexible polymeric films with unprecedented tunability of their photochromic properties that can be employed as rewritable multi-coloured devices.
\end{abstract}

\section{INTRODUCTION}

During the last decades both the industrial and the academic sectors have shown growing interest towards organic photochromes for the preparation of colour-tunable functional materials. ${ }^{1-3}$ Aiming to address specific applications, an increasing number of strategies are being developed to finely tailor the photochromic performance of these systems (e.g. switching colours, rates and wavelengths). This is the case of T-type photochromes, the capacity of which to undergo UV-induced colouration and subsequent spontaneous colour bleaching in the dark (i.e. direct photochromism) is currently employed for the fabrication of photoprotective coatings on industrial scale (e.g. in ophthalmic lenses and smart windows). ${ }^{3}$ However, a wealth of research is devoted to additionally accomplish reverse photochromism with these compounds (i.e. photoinduced colour fading followed by thermal coloration after irradiation), ${ }^{4,5}$ which would not only allow operation with less energetic and harmless visible light, but also grant access to novel applications, such as anticounterfeiting technologies, rewritable displays and optical data storage systems, among others. ${ }^{6-10}$ In spite of this, functional solid devices based on reverse T-type photochromes are very scarce and only started to be reported recently (e.g. in multicoloured light-responsive rewritable devices). ${ }^{11-17}$

Although new families of dyes can be prepared to directly exhibit reverse photochromism (e.g. imidazolebased compounds ${ }^{18-20}$ and donor-acceptor Stenhouse ad$\left.\operatorname{ducts}^{21}\right)$, much attention is dedicated to achieve this behaviour by means of well-known, commercially available and largely used T-type photochromes such as spirooxazines and spiropyrans. ${ }^{4,5}$ Normally, these compounds show direct photochromism since their thermodinamically stable form is the closed, colourless spiro state, which isomerizes to the coloured, unstable merocyanine (MC) when irradiated. ${ }^{1,3}$ However, several methodologies have been proposed to alter the relative stability of both isomers and attain reverse photochromism, ${ }^{6-17}$ where stabilization of the MC form is accomplished via: a) chemical modification of the dyes ${ }^{22-26}$ resulting in a dramatic variation of their electronic properties, ${ }^{27}$ intramolecular hydrogen-bond formation, ${ }^{23}$ or complexation with metals; ${ }^{28-}$ $\left.3^{36} \mathrm{~b}\right)$ dispersion of the dyes in selected media, ${ }^{37-40}$ which either stabilize the more polar zwitterionic MC structure (e.g. via hydrogen-bond interactions) or convert it into the protonated form $\mathrm{MCH}^{+}$through acid-base reactions. ${ }^{41^{-}}$ 43

All these strategies have been explored to obtain reverse photochromism with spirocompounds in liquid media (e.g. protic solvents, ${ }^{37-38}$ ionic liquids, ${ }^{44-45}$ solutions of acids $^{46-47}$ or metal ions ${ }^{28-36}$ ) as well as in bulk or micro/nanostructured solid systems, which are eventually more relevant for practical applications (e.g. polymers, ${ }^{26}$ polar silica, ${ }^{6,48-52}$ metal-organic framework, ${ }^{53}$ gold nanoparticles, ${ }^{54}$ hydrogels, ${ }^{55-56}$ liquid-filled capsules ${ }^{57}$ ). Nevertheless, the materials so far prepared in this way do not provide flexible tunability of their photochromic responses (i.e. colour in the dark and isomerization kinetics) since their properties are very dependent on the particular dyes and host matrices selected and, in some 
a)

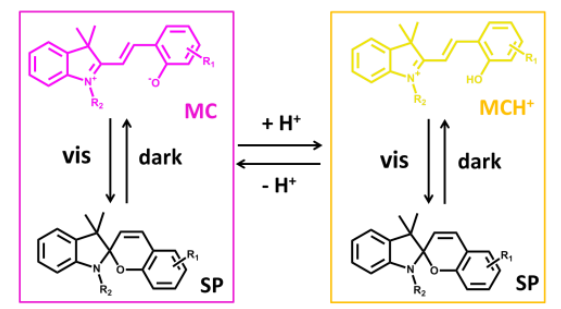

b)

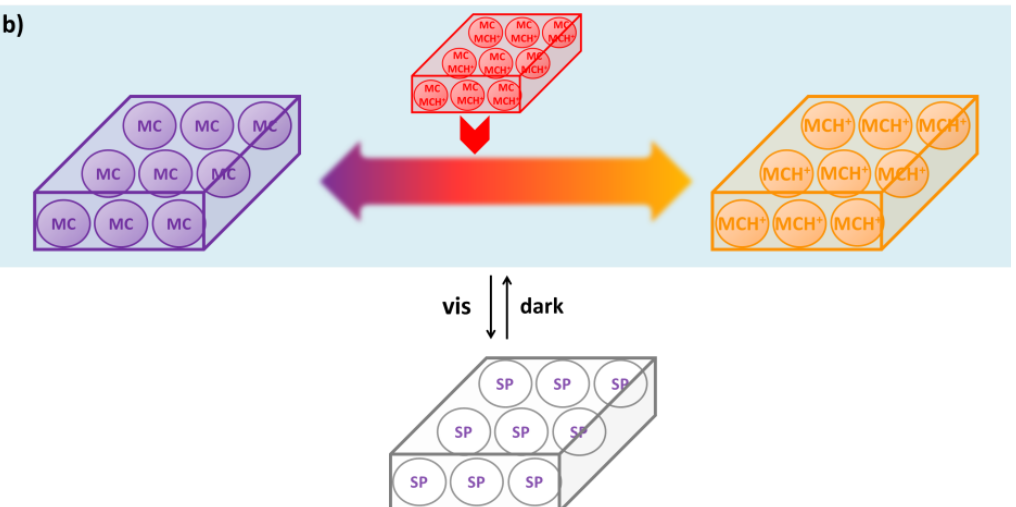

Scheme 1: Strategy used in this work to obtain tunable reverse photochromism with spiropyrans: a) modulation of the coloured merocyanine forms (non-protonated $\mathrm{MC}$ vs. protonated $\mathrm{MCH}^{+}$forms); $b$ ) encapsulation of the designed dye mixtures and integration of the core-shell capsules in solid films.

cases, even specific chemical modification of these species is needed. Therefore, the development of a simple approach to design and achieve tunable reverse photochromism in solid materials from readily available commercial dyes without requiring chemical derivatization would be highly desirable, thus paving the way for the broad use of this technology in real applications.

To reach this objective, in this work we propose a novel methodology that relies on: a) the embedment of readily accessible spiropyran dyes in rationally designed non-volatile media (with hydrogen-bonding and/or acidic properties), to fine control the equilibrium between the differently coloured merocyanine forms (typically, non-protonated, low-energy visible light absorbing MC and protonated, high-energy visible light absorbing $\mathrm{MCH}^{+}$forms ${ }^{4-43}$ ) and their relative concentration ratio; b) the encapsulation of these dye-containing mixtures in core-shell polymer capsules to preserve the photochromism in the solid state, as recently demonstrated by us, ${ }^{57}$ and c) the integration of these capsules in polymeric matrices, to achieve solid materials with high and straightforward tunability of the reverse photochromic performances (Scheme 1).

\section{RESULTS and DISCUSSION}

2.1. Tunable reverse photochromism of spiropyrans in nonanoic acid

In a first step, the capacity to tune the reverse photochromic behaviour of spiropyrans (SP) by modulating their acid-base properties was investigated in bulk solution. For this study we chose five commercially available SPs (see supporting information, Scheme S1), holding different chemical and electronic properties: 1',3',3'trimethyl-6-nitrospiro[1(2H)-benzopyran-2,2'-indoline (SP-1), 1',3'-dihydro-8-methoxy-1',3',3'-trimethyl-6nitrospiro[2H-1-benzopyran-2,2'-(2H)-indole] (SP-2), 1',3',3'-trimethylspiro[1(2H)-benzopyran-2,2'-indoline]

(SP-3), (6-bromo-1',3',3-trimethylspiro[1(2H)benzopyran-2,2'-indoline] (SP-4) and 8-methoxy-1,3',3'trimethylspiro[1(2H)-benzopyran-2,2'-indoline] (SP-5).
Importantly, these compounds differ on the basicity of the phenolate group of their MC isomers due to the distinct substituents that they present. As such, they could differently interact with nonanoic acid (NA), a weaklyacid non-volatile medium that we already proved to stabilize the open form of spiropyrans. ${ }^{57}$

When colourless SP-1-SP-5 were dissolved in NA (c = 14-18 $\mathrm{mM}$ ), different colours were effectively observed to develop in the dark, which we ascribed to stabilization of their merocyanine isomer as $\mathrm{MC}, \mathrm{MCH}^{+}$or an equilibrium mixture of both (Figure 1a). On one hand, brownyellow NA solutions characteristic of the protonated $\mathrm{MCH}^{+}$form ${ }^{41-43}$ were obtained for $\mathbf{S P}-3$ and $\mathbf{S P}_{-5}$ $\left(\lambda_{\text {abs, } \max }{ }^{\text {SP-3/NA }}=430 \mathrm{~nm}, \lambda_{\text {abs, } \max }{ }^{\text {SP-5/NA }}=400 \mathrm{~nm}\right)$, for which larger basicity is expected upon ring-opening due to the absence of an electron-withdrawing group (EWG) at the para position of the phenolate ring. On the other hand, SP-2 produced a violet solution in NA $\left(\lambda_{\mathrm{abs}, \max } \mathbf{S P}\right.$ ${ }^{2 / \mathrm{NA}}=564 \mathrm{~nm}$ ) with very little absorption in the region around $430 \mathrm{~nm}$, indicating selective formation of the non-protonated MC state as a consequence of the less basic character of its o-methoxy-p-nitrophenolate group. ${ }^{41-43}$ Finally, mixtures of the $\mathrm{MC}$ and $\mathrm{MCH}^{+}$forms were obtained for NA solutions of SP-1 and SP-4, which showed different spectral contributions from both species owing to their distinct acid-base properties: while major formation of MC was observed for SP-1 also bearing a low-basicity $p$-nitrophenolate group upon isomerization (red colour, $\lambda_{\text {abs, max }}{ }^{\text {SP-1/NA }}=528 \mathrm{~nm}$ with a pronounced shoulder at around $430 \mathrm{~nm}$ ), a much larger intensity of the $\mathrm{MCH}^{+}$absorption band was found for $\mathbf{S P}-\mathbf{4}$ due to the presence of a weaker, bromine EWG that insufficiently stabilizes the non-protonated MC state (yellow colour, $\lambda_{\mathrm{abs}, \max 1}{ }^{\text {SP-4/NA }}=437 \mathrm{~nm}$ and $\lambda_{\mathrm{abs}, \max 2}{ }^{\text {SP-4/NA }}=$ $550 \mathrm{~nm})$.

Notably, irradiation with visible light (laser pointer at $532 \mathrm{~nm}$ and $470 \mathrm{~mW} / \mathrm{cm}^{2}$, white-light emitting torch 
a)
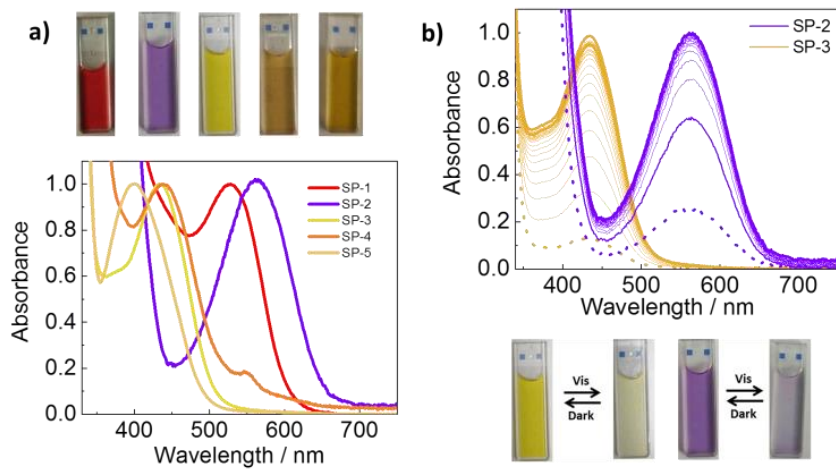

Figure 1: a) Digital images and absorption spectra of NA solutions of SP-1-SP-5. b) Digital images and absorption spectra of SP-2 and SP-3 in NA after irradiation with visible light (dashed line) and during recolouration in the dark.

with $\lambda_{\text {exc }}>400 \mathrm{~nm}$ and $20 \mathrm{~mW} / \mathrm{cm}^{2}$ ) provoked colour fading of all the NA solutions of SP-1-SP-5, a process that was reverted in the dark according to a first-order kinetics characteristic of photochrome backisomerization in homogeneous liquid systems $\left(k^{\mathrm{SP}-1 / \mathrm{NA}}=\right.$ $0.013 \mathrm{~min}^{-1}, k^{\mathrm{SP}-2 / \mathrm{NA}}=0.70 \mathrm{~min}^{-1}, k^{\mathrm{SP}-3 / \mathrm{NA}}=0.52 \mathrm{~min}^{-1}, k^{\mathrm{SP}-}$ ${ }_{4}^{4 / \mathrm{NA}}=0.51 \mathrm{~min}^{-1}, k^{\mathrm{SP}-5 / \mathrm{NA}}=6.1 \mathrm{~min}^{-1}$, Figure $\mathbf{1 b}$ and Figures S1-2). This, together with the different colours obtained for each of these solutions, demonstrate that tunable T-type reverse photochromism can be attained by simply dissolving commercial SP dyes in NA, without requiring further chemical derivatization or the use of additives.

\subsection{Colour tuning for individual spiropyran pho-} tochromes

On the basis of the results obtained in NA for different types of spiropyrans, tunable reverse photochromism could be reached for individual dyes if the equilibrium between their non-protonated and protonated merocyanine forms could be altered upon modification of the surrounding medium. To validate this hypothesis, we chose SP-1 as a benchmark system since it produces a reddish mixture of both $\mathrm{MC}$ and $\mathrm{MCH}^{+}$in NA, and we aimed to tune its colour in the dark by varying the relative concentration of these species using different solvents. In this study, well-designed, non-volatile solvents were used because they are more suitable for the fabrication of solid photochromic materials based on coreshell capsules.

According to the SP-1 behaviour in NA, predominant formation of the yellow $\mathrm{MCH}^{+}$or the violet $\mathrm{MC}$ forms of this dye should require increasing or decreasing the acidity of the media. Indeed, addition of a little amount of $n$-dodecylphosphonic acid (DPA) to a red solution of SP-1 in NA (DPA/NA molar ratio $=1 / 143$ ) turned the sample yellow $\left(\lambda_{\text {abs,max }}{ }_{\text {SP- } / / \mathrm{NA}-\mathrm{DPA}}=421 \mathrm{~nm}\right)$ and led to the disappearance of the absorption band at $\lambda_{\mathrm{abs}, \max }=528$ $\mathrm{nm}$, thus suggesting quantitative protonation of MC (Figures 2a).
On the other hand, when SP-1 was dissolved in a nonacidic protic oil such as dihydroxy-terminated poly(dimethylsiloxane-co-diphenylsiloxane) (PDMS$\mathrm{OH})$, it was selectively stabilized as its violet MC form through hydrogen bonds $\left(\lambda_{\max }{ }^{\text {SP-1/PDMS-OH }}=551 \mathrm{~nm}\right.$, Figure 2a) and no absorption contribution from $\mathrm{MCH}^{+}$at around $430 \mathrm{~nm}$ was detected. In both cases, reverse photochromism was preserved since the two SP-1 solutions bleached when subjected to visible light irradiation and recoloured in the dark (Figures 2b-d). Noticeably, different back-isomerization rate constants were measured for these samples and that in NA $\left(k^{\text {SP-1/NA-DPA }}=0.0044\right.$ $\min ^{-1}, k^{\text {SP-1/PDMS-OH }}=0.037 \mathrm{~min}^{-1}$ ), which indicates that not only the colour but also the switching kinetics of photochromes can be modulated with the strategy introduced in this work (Figure $\mathbf{S}_{\mathbf{3}}$ ).

Once demonstrated colour-tunable reverse photochromsim for SP-1, we explored the generality of this behaviour for other spiropyrans. In particular, our attention focused on dyes producing merocyanine states with different basicity than SP-1, since this is a crucial parameter controlling the equilibrium between their MC and $\mathrm{MCH}^{+}$forms.
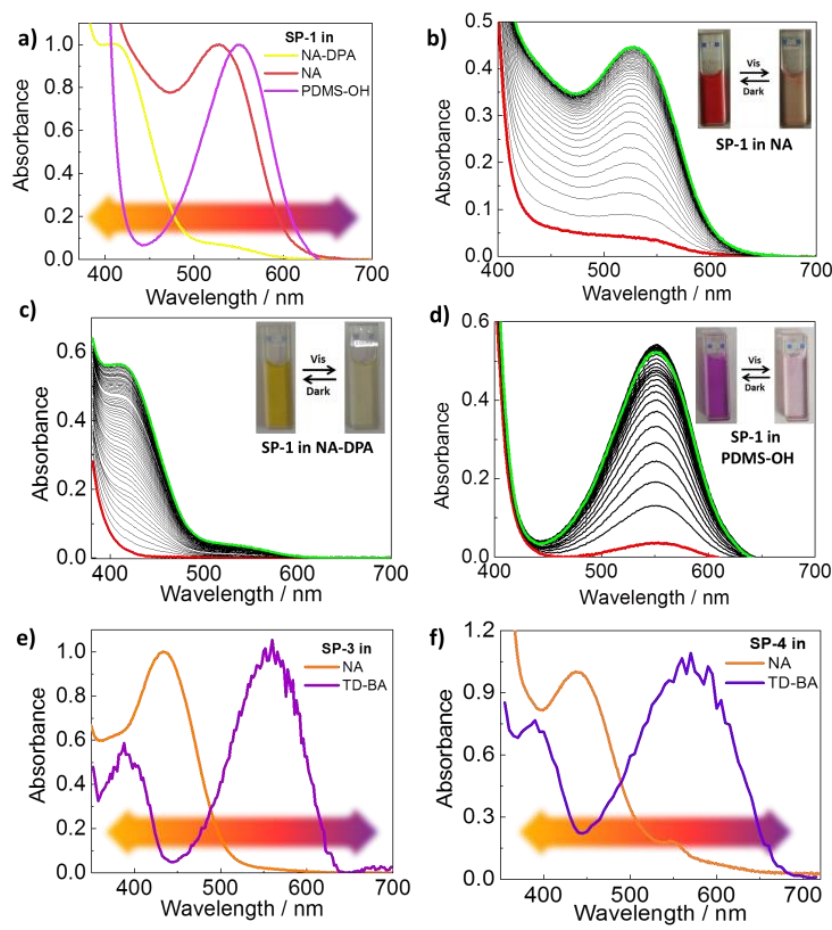

Figure 2: a) Absorption spectra of SP-1 in NA-DPA, NA and PDMS-OH; Absorption spectra of SP-1 in b) NA, c) NADPA and d) PDMS-OH in the dark (green line), after irradiation with visible light (red line) and during thermal recolouration (black lines); Absorption spectra of e) SP-3 and $f$ ) SP-4 in NA and TD-BA.

For this reason, additional studies were conducted for SP-3 and SP-4, which represent high- and intermediate basicity cases in comparison to nitrosubstituted SP-1, respectively. Actually, for both dyes, dissolution in weak acid NA gave rise to predominant formation of $\mathrm{MCH}^{+}$, in 
contrast to SP-1 (see Figure 1a). In order to favour stabilization of the non-protonated MC form of SP-3 and SP4, we employed a solid mixture made of tetradecanol (TD) and bisphenol A (BA) (TD/BA molar ratio = 18/1), which is generally used in thermochromic materials to stabilize the open form of spirolactones through hydrogen-bond interactions..$^{5-59}$ The dissolution of SP-3 and SP-4 in the TD-BA mixture provoked the development of a pink/violet colour and the rise of absorption bands at around $390 \mathrm{~nm}$ and $565 \mathrm{~nm}$ (Figures 2e-f), thereby indicating MC formation. In both cases, reverse photochromism was observed upon visible-light irradiation with relatively large back-isomerization rate constants in the dark despite the solid nature of the solvent used $\left(k^{\text {SP-3/TD-BA }}=1.7 \mathrm{~min}^{-1}\right.$ and $k^{\text {SP-4/TD-BA }}=0.70 \mathrm{~min}^{-1}$, Figure S4). Interestingly, the growth of both absorption bands at $390 \mathrm{~nm}$ and $565 \mathrm{~nm}$ took place with very similar kinetics in these experiments, which further suggests that they derive from the same MC species rather than from a mixture of the protonated and non-protonated merocyanine forms of SP-3 and SP-4 (Figure $\mathbf{S}_{5}$ ).

2.3. Core-shell capsules with tunable reverse photochromism

As shown above, full tunability of reverse photochromism can be achieved in bulk solution for a wide range of commercially available spiropyran dyes by simple solvent variation. However, direct transfer of this behaviour to the solid state is required for the fabrication of functional photochromic materials such as inks or paints. Exploiting the non-volatility of the solvents employed, we aimed to achieve this objective by preparing stable core-shell capsules, a strategy that we have already proven to allow preservation of bulk photochromic $^{57,60}$ and/or thermochromic ${ }^{61}$ properties in solid matrices.

In view of this, polymer-coated capsules of solutions of SP-1 in NA-DPA, NA and PDMS-OH, and of SP-3 and SP-4 in NA and TD-BA were prepared through the reaction-free emulsion-solvent evaporation method (see the Experimental section and Supporting Information for the detailed procedure), which prevents dye degradation by undesired chemical reactions. ${ }^{62}$ After optimization, poly(methylmethacrylate) (PMMA), polyethersulfone (PES) and poly(bisphenol A carbonate) (PC) were employed as shell polymers depending on the encapsulated mixture. SEM images showed the successful formation of the capsules loaded with SP-1 (SP-1/NADPA@PMMA, SP-1/NA@PMMA, SP-1/PDMSOH@PMMA), SP-3 (SP-3/NA@PMMA, SP-3/TDBA@PC) and SP-4 solutions (SP-4/NA@PMMA, SP4/TD-BA@PES), the sizes of which ranged from 1-5 $\mu \mathrm{m}$ (for NA, NA-DPA) to 20-30 $\mu \mathrm{m}$ (PDMS-OH and TD-BA systems, Figure 3). ${ }^{1} \mathrm{H}-\mathrm{NMR}$ spectroscopy proved the encapsulation of NA (47-52\% wt.), PDMS-OH ( $54 \%$ wt.) and TD (47-57 wt. \%, Figures S6-S7). For NA and TD, similar melting points $\left(T_{m}\right)$ were measured for the capsules and in bulk by DSC $\left(T_{m}{ }^{\mathrm{NA}}=8-12.5^{\circ} \mathrm{C},{ }^{63} T_{m}{ }^{\mathrm{NA}-\mathrm{DPA}}=4\right.$ ${ }^{\circ} \mathrm{C}$ and $\left.T_{m}{ }^{\mathrm{TD}}=33.5-35.9{ }^{\circ} \mathrm{C}^{63}\right)$, which further supports that these materials are not mixed with the shell polymer and therefore are essentially confined to the core of the capsules (Figure S8). ${ }^{64}$ Consequently, this should guarantee preservation of the bulk photochromic properties of the loaded dyes if they remain in the coreforming media. Indeed, the freeze-dried capsules of the SP-1 mixtures showed yellow (SP-1/NA-DPA@PMMA), red (SP-1/NA@PMMA) and violet (SP-1/PDMSOH@PMMA) colours as well as reflectance-mode absorption spectra $\left(\lambda_{\mathrm{abs}, \max }=425,528\right.$ and $550 \mathrm{~nm}$, respectively) that nicely reproduced the optical properties of the bulk solutions (Figure 3). Similar results were obtained for SP-3/NA@PMMA and SP-4/NA@PMMA (yellow colour, $\lambda_{\text {abs, max }}=434$ and $440 \mathrm{~nm}$, respectively) and SP-3/TD-BA@PC and SP-4/TD-BA@PES (pinkviolet colour, $\lambda_{\mathrm{abs}, \max }=390$ and $554 \mathrm{~nm}$ and $\lambda_{\mathrm{abs}, \max }=388$ and $546 \mathrm{~nm}$, respectively), which demonstrates that encapsulation of dye-solvent mixtures in polymer coreshell capsules does not alter the stabilization mechanisms and efficiencies of the merocyanine state of the Ttype photochromes considered (Figure 3 and Table $\mathbf{S} \mathbf{1}$ ).

In addition, irradiation with visible light provided in all the cases colourless capsules, which recoloured in the dark with rather similar rates to the bulk systems $\left(k^{\mathbf{S P}-}\right.$ 1/NA-DPA@PMMA $=0.0038 \mathrm{~min}^{-1}, k^{\mathrm{SP}-1 / \mathrm{NA} @ P M M A}=0.012 \mathrm{~min}^{-1}$, $k^{\mathrm{SP}-1 / \mathrm{PDMS}-\mathrm{OH} @ \mathrm{PMMA}}=0.042 \mathrm{~min}^{-1}, k^{\mathrm{SP}-3 / \mathrm{NA} @ \mathrm{PMMA}}=0.17 \mathrm{~min}^{-1}$, $k^{\mathrm{SP}-4 / \mathrm{NA} @ \mathrm{PMMA}}=0.16 \mathrm{~min}^{-1}, k^{\mathrm{SP}-3 / \mathrm{TD}-\mathrm{BA} @ \mathrm{PC}}=0.72 \mathrm{~min}^{-1}, k^{\mathrm{SP}-}$ 4/TD-BA@PES $\left.=0.19 \mathrm{~min}^{-1}\right)($ Figure 3 and Figures S9-11, Table $\mathrm{S}_{2}$ ). For SP-3- and SP-4-containing capsules, however, we systematically measured slower recolouration kinetics than in bulk mixtures, which was tentatively ascribed to the formation of rigid photochromic subdomains in the polymer shell where the isomerization processes are slightly hampered. In spite of this, the fast colour recovery rates of these capsules allowed fatigue resistance studies to be performed, which confirmed photochrome stability after a few cycles of light-induced decolouration and recolouration in the dark (Figure S12).

\subsection{Photochromic films and rewritable devices}

Core-shell capsules displaying tunable reverse photochromism were finally dispersed in polymeric matrices to allow the fabrication of composite materials that could be of relevance for practical applications. For this, capsule-containing, flexible poly(vinylalcohol) (PVA) and polyacrylamide (PA) films were prepared by dropcasting an aqueous suspension of these water-soluble polymers and of the capsules of choice in a Petri plate, from which they could be easily peeled off after solvent evaporation. For the preparation of the films, the capsules obtained from SP-1 and $\mathbf{S P}-\mathbf{4}$ in different media (NA, NA-DPA, PDMS-OH and TD-BA) were used, which maintained their shape and structure once embedded in the polymer matrices (Figure S13). As expected, the resulting polymer films were all coloured in the dark and preserved the optical properties and reverse photochromic behaviour of the capsules dispersed (Figures 
S14-15, Tables S1-S2). Notably, from each photochromic dye (i.e. SP-1 and SP-4), two or more differently coloured films could be prepared, thus demonstrating the capability of the methodology introduced in this work to provide fine tunability of reverse photochromism for the fabrication of functional materials (Figure 4).
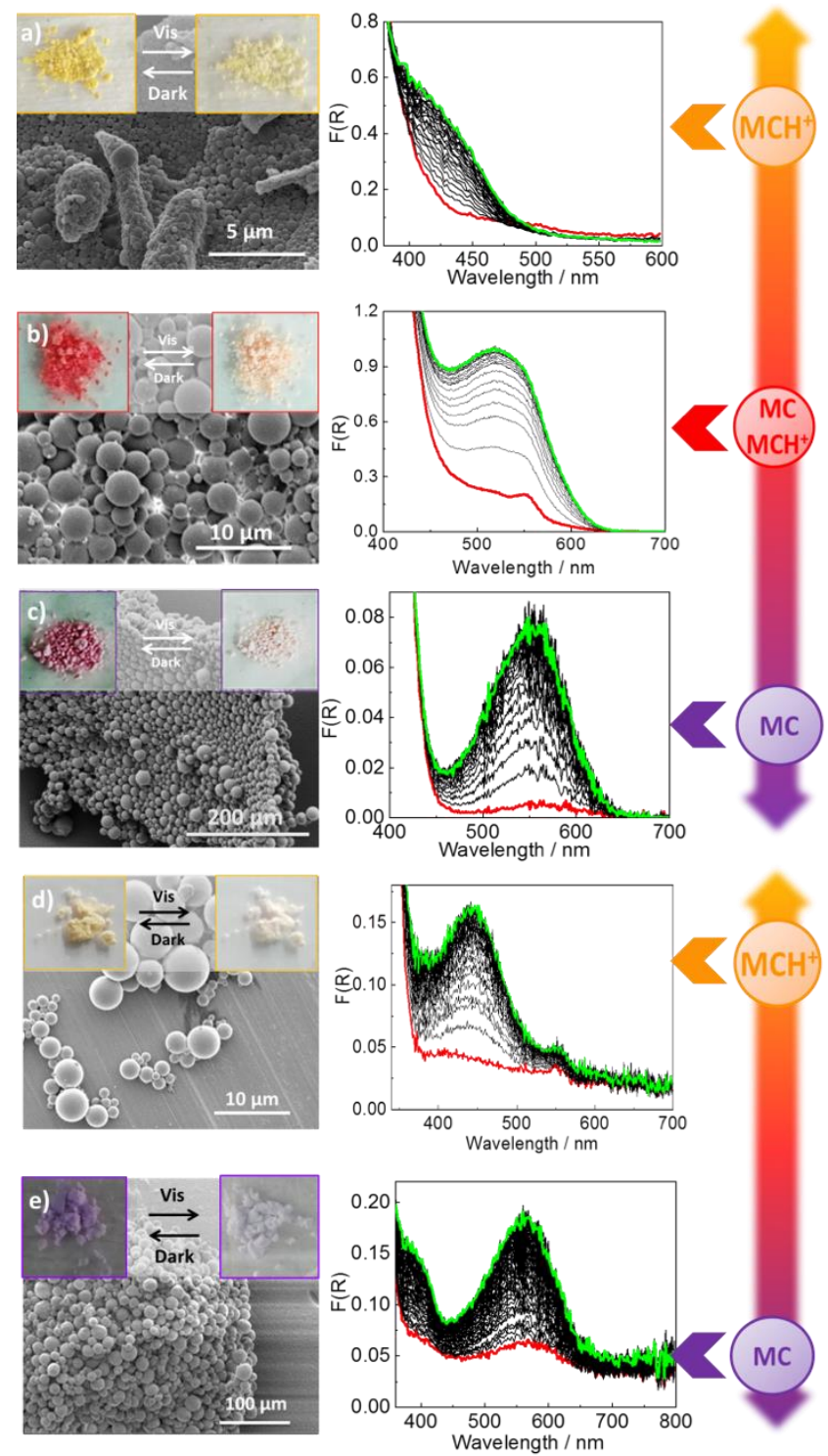

Figure 3: SEM images and reverse photochromic behaviour of: a) SP-1/NA-DPA@PMMA, b) SP-1/NA@PMMA, c) SP-1/PDMS-OH@PMMA, d) SP-4/NA@PMMA and e) SP4/TD-BA@PES microcapsules. For each sample, reflectance-mode absorption spectra are given in the dark (green line), after irradiation with visible light (red line) and during thermal recolouration (black lines).

As a proof of this concept, we explored the use of films containing SP-1 and SP-4-loaded capsules as rewritable papers. Noticeably, by using just one single photochromic dye (e.g. SP-1), multicoloured films could be written and erased several times with different black masks or by hand-writing with a 532-laser pointer, obtaining writings and draws with very good contrast and spatial resolution (Figure 5).

\section{CONCLUSIONS}

In summary, we reported in this work a novel, reactionfree, universal and straightforward strategy to achieve tunable reverse photochromism in solid functional materials. By exploiting the capacity of commercially available spiropyrans to produce different stable merocyanine forms with distinctive optical properties, we demonstrated that distinct colours and switching rates could be obtained for these photochromes without the need of chemical derivatization by simply varying the nature of the surrounding solvent. In addition, we showed that this behaviour could be directly transferred to solid matrices by preparing polymer capsules loaded with spiropyran solutions and eventually dispersing them in the final material of interest. As a result, polymer films with up to 3 different photochromic responses (i.e. colours and isomerization kinetics) could be generated from the same commercial dye, providing unprecedented tunability of the photochromic properties in the solid state. Considering that more colours could be obtained by combining capsules of different types, which might also display thermochromic, halochromic and/or fluorescence behaviour, functional materials could be prepared from spiropyran dyes exhibiting multicolour and multistimuli responses. 


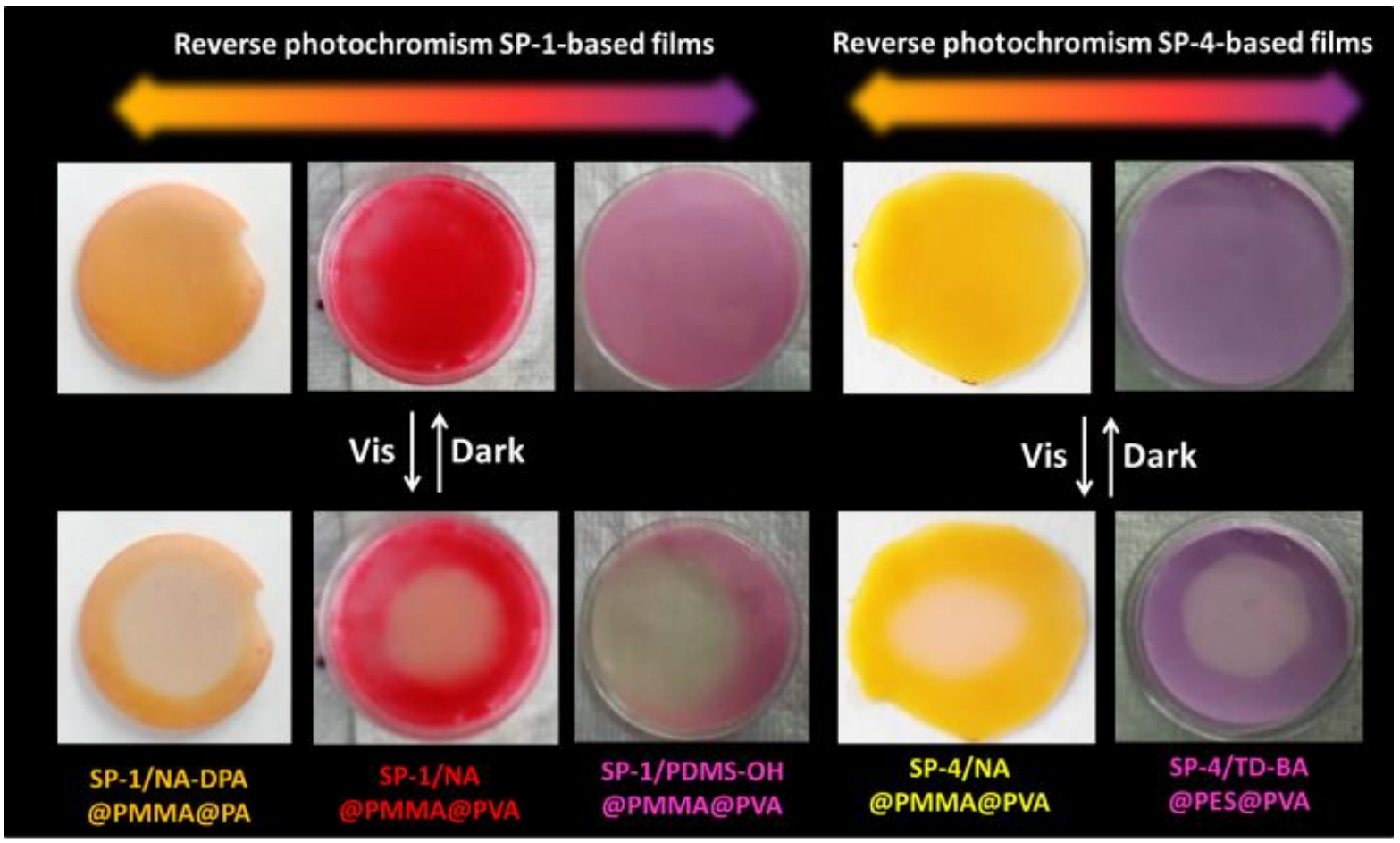

Figure 4: PVA and PA films obtained from SP-1- and SP-4-loaded capsules with different core materials, which display colour-tunable reverse photochromism.

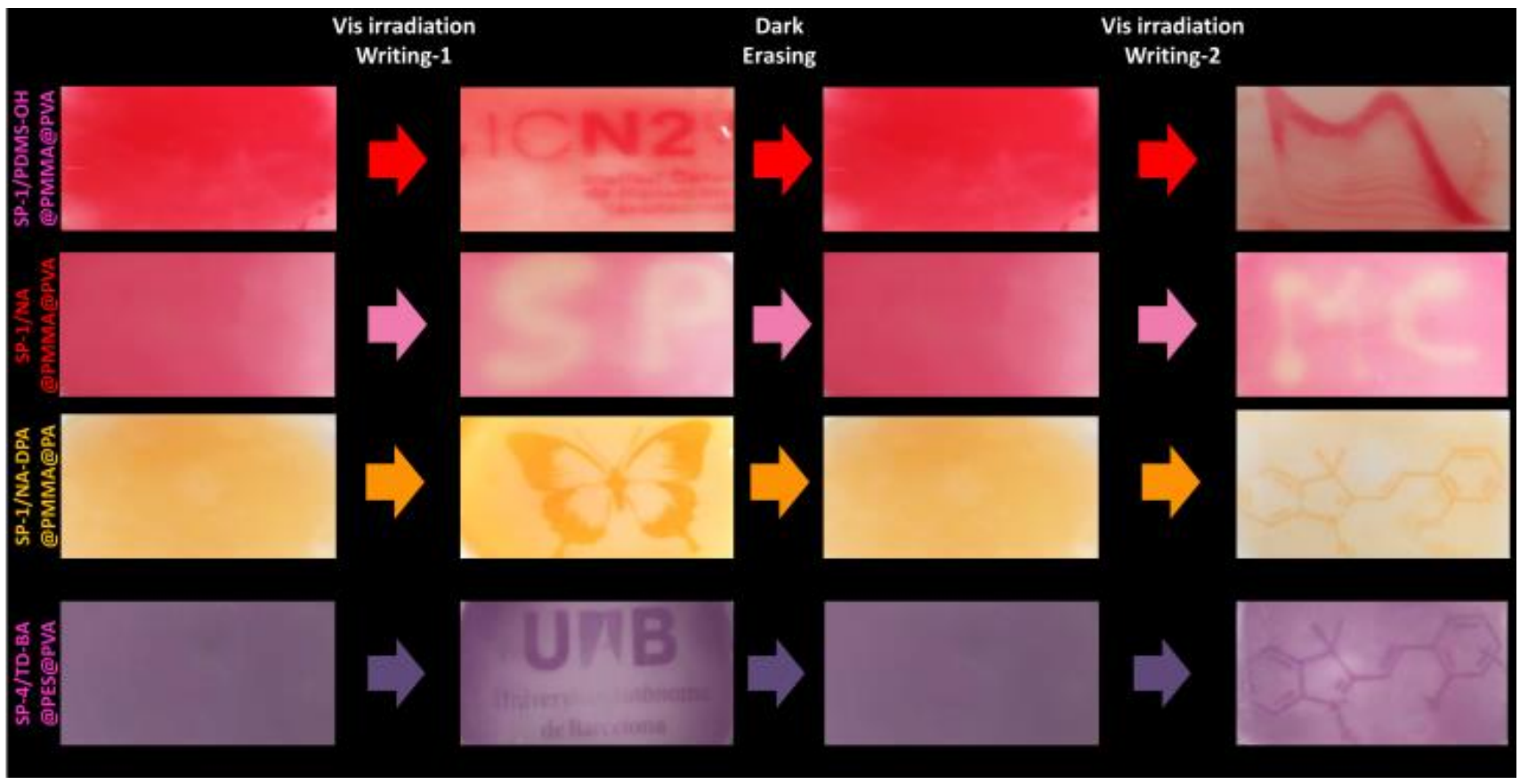

Figure 5: Writing/erasing/rewriting of PVA (or PA) films of SP-1 and SP-4 capsules containing different media and providing different colours (hand-writing with laser pointer irradiation, $470 \mathrm{~mW} / \mathrm{cm}^{2}$, or printed-like drawing with visible light torch, $20 \mathrm{~mW} / \mathrm{cm}^{2}$, in the presence of a black mask). 


\section{EXPERIMENTAL SECTION}

\section{Materials}

The polymers used for the preparation of the capsules, poly(methylmethacrylate) (PMMA, MW = 120000), poly(bisphenol-A carbonate) $(\mathrm{PC}, \mathrm{MW}=45000)$, polyethersulfone (PES, MW = 2200o)) and the films (poly(vinyl alcohol) (PVA, Mowiol ${ }^{\circledR}$ 4-88, 99\% hydrolyzed, medium molecular weight), polyacrylamide (PA)), and the colour developers (bisphenol-A (BA, 99\%)) and dihydroxyterminated poly(dimethylsiloxane-co-diphenylsiloxane) (PDMS-OH) were purchased from Sigma Aldrich. Nonanoic acid (NA, 97\%), 1-tetradecanol (TD, 97\%) and ndodecylphosphonic acid (DPA, 95\%) were acquired from Alfa Aesar. Photochromes 1,3',3'-trimethyl-6nitrospiro[1(2H)-benzopyran-2,2'-indoline (SP-1), 1',3'dihydro-8-methoxy-1',3',3'-trimethyl-6-nitrospiro[2H-1-

benzopyran-2,2'-(2H)-indole] (SP-2), 1',3',3'trimethylspiro[1(2H)-benzopyran-2,2'-indoline] (SP-3), (6-bromo-1',3',3'-trimethylspiro[1(2H)-benzopyran-2,2'indoline $\quad($ SP-4) and 8-methoxy-1,3',3'trimethylspiro[1 $(2 \mathrm{H})$-benzopyran-2,2'-indoline] (SP-5) were all obtained from TCI chemicals. $\mathrm{CH}_{2} \mathrm{Cl}_{2}$ was acquired from Scharlau. All commercial solvents and chemicals were used without any further purification. Distilled water was used in all syntheses. Deuterated solvents were acquired from Euriso-Top.

\section{Characterization}

Electron microscopy (EM): Scanning electron microscopy (SEM) images were collected on a scanning electron microscope FEI Quanta 650 FEG at acceleration voltages between 2-10 kV. Samples were mounted on SEM metal stubs covered with aluminum or carbon tape and they were coated with a thin layer of platinum $(\sim 5$ $\mathrm{nm})$. Microcapsules suspensions $(100 \mu \mathrm{L})$ were deposited on the stub by drop casting, allowing solvent evaporation in air at room temperature. For solid powders, approximately $1 \mathrm{mg}$ of capsules was deposited on the adhesive carbon tape.

Proton nuclear magnetic resonance ( $\left.{ }^{1} H-N M R\right)$ : 20-30 mg of microcapsules were dissolved in $\mathrm{CDCl}_{3}$ and $\mathrm{i}_{\mathrm{H}} \mathrm{H}$ NMR spectra were recorded using the following spectrometers: Bruker DPX250 (250 MHz for ${ }^{1} \mathrm{H}-\mathrm{NMR}$ ) and Bruker DPX40o (40o MHz for $\left.{ }^{1} \mathrm{H}-\mathrm{NMR}\right)$. The spectra are given in chemical shifts, $\delta(\mathrm{ppm})$. The peaks are defined as singlets $(\mathrm{s})$, triplets $(\mathrm{t})$ or multiplets $(\mathrm{m}) .10 \mu \mathrm{l}$ of DMF $\left(\delta=2.88\left(\mathrm{~s},{ }_{3} \mathrm{H}, \mathrm{CH}_{3}\right), 2.97\left(\mathrm{~s}, 3 \mathrm{H}, \mathrm{CH}_{3}\right), 7.96\left(\mathrm{~s},{ }_{1} \mathrm{H}, \mathrm{CH}\right)\right)$ were added to the samples, as an internal reference, for quantitative determination of the microcapsules payload.

Absorption spectra: The absorption spectra of the solid and liquid samples were obtained from a Cary 6o spectrophotometer. Liquid solutions were measured in transmittance mode. Solid samples were measured through diffuse reflectance mode, using an external integrating sphere connected with a fiber optic. As a reference, the same material (i.e. TD, capsules, films) without the dye, was used. The absorption spectra were estimated from the measured diffuse reflectance spectra through the Kubelka-Munk equation $\left(F(R)=(1-R)^{2} / 2 R\right.$ )$^{65}$ Time-dependent absorption measurements were performed using the spectrophotometer in scan mode. For all the photochromic materials, their thermal backisomerization was monitored by recording the absorption (or reflectance) spectra at different time delays, in the dark after reaching their photostationary state.

Differential Scanning Calorimetry (DSC): Measurements were carried out in a TA Instruments Q20 differential scanning calorimeter. Approximately between 4-7 mg of a sample were deposited on a $0.5 \mathrm{~cm}$-in-diameter aluminum pan. An empty pan was used as a reference. The scanning rate was $10 \mathrm{~K} / \mathrm{min}$ for both the heating and cooling processes. The scanned temperature range depended on the sample. The melting/crystallization point were calculated from the intersection between two tangents (one is the slope of the endo- or exothermic peak and the other is the straight line of the baseline).

\section{Irradiation methods}

Visible light irradiations were carried out by using a green laser pointer (JD850, $532 \mathrm{~nm}, 470 \mathrm{~mW} / \mathrm{cm}^{2}$ ) or a non-coherent flashlight torch (UltraFire CREE XMLT6, $20 \mathrm{~mW} / \mathrm{cm}^{2}$ ).

\section{Synthesis of core-shell capsules}

All capsules were prepared through the solvent evaporation method. ${ }^{62}$ Typically the polymer (PMMA, PC, PES, 0.25-0.75 g) was dissolved in $\mathrm{CH}_{2} \mathrm{Cl}_{2}$ (5 mL) upon magnetic stirring, while the photochrome of interest (SP-1-SP-5, 10-30 mg) was dissolved in the corresponding colour developing mixture (0.5-1.o g). During photochrome dissolution, colour development was observed. Once all the photochrome was dissolved, the obtained photochromic mixture was poured into the $\mathrm{CH}_{2} \mathrm{Cl}_{2}$ solution. The final organic mixture was subsequently added to a previously prepared PVA water solution (10 $\mathrm{mL}, 1$ wt. \%). The mixture was homogenized (T18 UltraTurrax $^{\circledast}$ IKA, 3000-5000 rpm) at room temperature for 10 minutes to induce the formation of the oil-in-water emulsion. Successively, the whole emulsion was transferred into a round bottom flask and was heated at $44^{\circ} \mathrm{C}$ under vacuum for 20 minutes, during which $\mathrm{CH}_{2} \mathrm{Cl}_{2}$ evaporated and the polymer precipitated around the non-volatile organic droplets. The obtained suspension was diluted with water $(30 \mathrm{~mL})$ and centrifuged at $4000-$ $10000 \mathrm{rpm}$ during 10 minutes. This process was repeated three times redispersing the capsules with water (10 $\mathrm{mL}$ ). After freeze-drying the resulting suspension in the dark, a coloured, fine solid powder was obtained. See the supporting information for the detailed amount of the components used in each capsule synthesis.

\section{Preparation of polymer films}

A fraction of the obtained suspension of the capsules ( $3 \mathrm{~mL}$ ) was dispersed homogeneously with the vortex in a previously prepared film-forming polymer solution 
(PVA or PA, 2-20 wt. \%). This solution was slowly transferred to a plastic Petri plate (diameter of $5.5 \mathrm{~cm}$ ) and water was allowed to evaporate on a flat surface at room temperature during 24-48 h. The final film was easily removed from the Petri plate once it was dry. See the supporting information for the detailed amount of the components used in each film.

\section{ASSOCIATED CONTENT}

Supporting Information. The Supporting Information include the list of materials used and respective providers, scheme of encapsulation process, chemical structures of used spiropyrans, procedures for the syntheses of the capsules, preparation of the films, descriptions of the characterization techniques, ${ }^{1} \mathrm{H}-\mathrm{NMR}$ spectra of dissolved capsules, DSC curves of capsules, kinetic curves for recolouration processes, further absorption spectra and digital images of bulk solutions, capsules and films, tables summarizing the absorption maxima and kinetic rates of the investigated systems. This material is available free of charge via the Internet at http://pubs.acs.org.

\section{AUTHOR INFORMATION}

\section{Corresponding Author}

Claudio Roscini

Catalan Institute of Nanoscience and Nanotechnology (ICN2), CSIC and The Barcelona Institute of Science and Technology (BIST), Campus UAB, Bellaterra, Barcelona o8193, Spain

\section{mail: Claudio.roscini@icn2.cat}

\section{Author Contributions}

The manuscript was written through contributions of all authors. / All authors have given approval to the final version of the manuscript.

\section{Funding Sources}

MAT2015-70615-R and CTQ2015-65439-R from MINECO/FEDER and 2017SGRoo465 from Generalitat de Catalunya. ICN2 acknowledges support from the Severo Ochoa (MINECO, Grant No. SEV-2017-0706) and the CERCA programmes (Generalitat de Catalunya).

\section{ACKNOWLEDGMENT}

This work was supported by projects MAT2015-70615-R and CTQ2015-65439-R from MINECO/FEDER and 2017SGRoo465 from Generalitat de Catalunya. ICN2 acknowledges support from the Severo Ochoa (MINECO, Grant No. SEV-2017-0706) and the CERCA programmes (Generalitat de Catalunya).

\section{ABBREVIATIONS}

$T_{m}$, melting point; DSC, differential scanning calorimetry; ${ }^{1} \mathrm{H}-\mathrm{NMR}$, proton nuclear magnetic resonance spectroscopy; UV-Vis, ultraviolet-visible; SEM, scanning electron microscopy; PVA, poly(vinyl alcohol); PMMA, poly(methylmethacrylate); PES, polyethersulfone; PC, poly(bisphenol A carbonate); PA polyacrylamide; NA, nonanoic acid; TD, tetradecanol; DPA, dodecylphosphonic acid; PDMS-OH, dihydroxy-terminated poly(dimethylsiloxane-co-diphenylsiloxane); BA, bisphenol A; SP-X, spiropyrans 1-5 (structures in supporting information); $\mathrm{MC}$, non-protonated merocyanine form, $\mathrm{MCH}^{+}$, protonated merocyanine; SP-X/YY@WW, capsules with shell made of WW, containing a mixture of spiropyran SP$\mathrm{X}$ in the color developing mixture YY; SP-X/YY@WW@KK, capsules in films of polymer KK.

\section{REFERENCES}

(1) Bertleson, R. C.; Maeda, S.; Van Gemert, B. Organic photochromic and thermochromic compounds, Vol. 1; Crano, J. C.; Guglielmetti, R. J. Eds.; Plenum: New York, 1999.

(2) Irie, M. Photochromism: Memories and SwitchesIntroduction Chem. Rev. 20oo, 100, 1683-1684.

(3) Corns, S. N.; Partington, S. M.; Towns, A. D. Industrial organic photochromic dyes Color. Technol. 2009, 125, 249-261.

(4) Barachevsky, V. A. Advances in photonics of organic photochromism J. Photochem. Photobiol., A 2018, 354, 61-69.

(5) Aiken, S.; Edgar, R. J. L.; Gabbutt, C.; Heron, B. M. Negatively Photochromic Organic Compounds: Exploring the Dark Side Dyes Pigm. 2018, 149, 92-121.

(6) Klajn, R. Spiropyran-Based Dynamic Materials. Chem. Soc. Rev. 2014, 43, 148-184.

(7) Pardo, R.; Zayat, M.; Levy, D. Photochromic OrganicInorganic Hybrid Materials Chem. Soc. Rev. 2011, 40, 672687.

(8) Pischel, U. Chemical Approaches to Molecular Logic Elements for Addition and Subtraction Angew. Chem. Int. Ed. 2007, 46, 4026-4040.

(9) Gentili, P. L. The Fundamental Fuzzy Logic Operators and some Complex Boolean Logic Circuits Implemented by the Chromogenism of a Spirooxazine Phys. Chem. Chem. Phys. 2011, 13, 20335-20344.

(10) Gentili, P. L. The Fuzziness of a Chromogenic Spirooxazine Dyes Pigm. 2014, 110, 235-248.

(11) Khazi, M. I.; Jeong, W.; Kim, J.-M. Functional Materials and Systems for Rewritable Paper Adv. Mater. 2018, 30, 1705310 .

(12) Wang, W.; Liu, L.; Feng, J.; Yin, Y. Photocatalytic Reversible Color Switching Based on Titania Nanoparticles Small Methods 2018, 1700273.

(13) Gao, Z.; Liu, L.; Tian, Z.; Feng, Z.; Jiang, B.; Wang, W. Fast-Response Flexible Photochromic Gels for SelfErasing Rewritable Media and Colorimetric Oxygen Indicator Applications ACS Appl. Mater. Interfaces 2018, 10, 33423-33433.

(14) Zhang, T.; Sheng, L.; Liu, J.; Ju, L.; Li, J.; Du, Z.; Zhang, W.; Li, M.; Zhang, S. X.-A. Photoinduced Proton Transfer between Photoacid and $\mathrm{pH}$-Sensitive Dyes: Influence Factors and Application for Visible-Light-Responsive Rewritable Paper Adv. Funct. Mater. 2018, 28, 1705532.

(15) Petriashvili, G.; De Santo, M. P.; Devadze, L.; Zurabishvili, T.; Sepashvili, N.; Gary, R.; Barberi, R. Rewritable Optical Storage with a Spiropyran Doped Liquid Crystal Polymer Film Macromol. Rapid Commun. 2016, 37, 500-505.

(16) Abdollahi, A.; Sahandi-Zangabad, K.; RoghaniMamaqani, H. Rewritable Anticounterfeiting Polymer Inks Based on Functionalized Stimuli-Responsive Latex Particles Containing Spiropyran Photoswitches: Reversible Photopatterning and Security Marking ACS Appl. Mater. Interfaces 2018, doi: 10.1021/acsami.8b14865.

(17) Abdollahi, A.; Sahandi-Zangabad, K.; RoghaniMamaqani, H. Light-Induced Aggregation and Disaggregation of Stimuli-Responsive Latex Particles Depending 
on Spiropyran Concentration: Kinetics of Photochromism and Investigation of Reversible Photopatterning Langmuir 2018, 34, 13910-13923.

(18) Yamaguchi, T.; Kobayashi, Y.; Abe, J. Fast Negative Photochromism of 1,1'-Binaphthyl-Bridged PhenoxylImidazolyl Radical Complex J. Am. Chem. Soc. 2016, 138, 906-913.

(19) Hatano, S.; Horino, T.; Tokita, A.; Oshima, T.; Abe, J. Unusual Negative Photochromism via a Short-Lived Imidazolyl Radical of 1, 1'-Binaphthyl-Bridged Imidazole Dimer J. Am. Chem. Soc. 2013, 135, 3164-3172.

(20) Yonekawa, I.; Mutoh, K.; Kobayashi, Y.; Abe, J. IntensityDependent Photoresponse of Biphotochromic Molecule Composed of a Negative and a Positive Photochromic Unit J. Am. Chem. Soc. 2018, 140, 1091-1097.

(21) Helmy, S.; Leibfarth, F. A.; Oh, S.; Poelma, J. E.; Hawker, C. J.; de Alaniz, J. R. Photoswitching Using Visible Light: a New Class of Organic Photochromic Molecules J. Am. Chem. Soc. 2014, 136, 8169-8172.

(22) Winkler, J. D.; Deshayes, K. Photodynamic Macrocycles J. Am. Chem. Soc. 1987, 109, 2190-2191.

(23) Inoue, E.; Kokado, H.; Shimizu, I.; Kobayashi, H.; Takakashi, Y. Photo-Decoloration Process of the Reverse Photochromic Spirans Bull. Chem. Soc. Jpn. 1972, 45, 19511956.

(24) Vallet, J.; Micheau, J.-C.; Coudret, C. Switching a pH Indicator by a Reversible Photoacid: a Quantitative Analysis of a New Two-Component Photochromic System Dyes Pigm. 2016, 125, 179-184.

(25) Chen, H.; Liao, Y. Photochromism based on Reversible Proton Transfer J. Photochem. Photobiol., A 2015, 300, 2226.

(26) Shimizu, I.; Kokado, H.; Inoue, E. Photoreversible photographic systems. VI. Reverse Photochromism of 1, 3, 3Trimethylspiro [Indoline-2, 2'-Benzopyran]-8'-Carboxylic Acid Bull. Chem. Soc. Jpn. 1969, 42, 1730-1734.

(27) Koelsch, C.F.; Workman, W.R. Some Thermochromic Spirans J. Am. Chem. Soc. 1952, 74, 6288-6289.

(28) Winkler, J. D.; Deshayes, K.; Shao B. Photodynamic Transport of Metal Ions J. Am. Chem. Soc. 1989, 111, 769770.

(29) Zhang, C.; Zhang, Z.; Fan, M.; Yan, W. Positive and Negative Photochromism of Novel Spiro [IndolinePhenanthrolinoxazines] Dyes Pigm. 2oo8, 76, 832-835.

(30) Winkler, J. D.; Bowen, C. M.; Michelet, V. Photodynamic Fluorescent Metal Ion Sensors with Parts Per Billion Sensitivity J. Am. Chem. Soc. 1998, 120, 3237-3242.

(31) Kopelman, R. A.; Snyder, S. M.; Frank, N. L. Tunable Photochromism of Spirooxazines via Metal Coordination J. Am. Chem. Soc. 2003, 125, 13684-13685.

(32) Wojtyk, J. T. C.; Kazmaier, P. M.; Buncel, E. Modulation of the Spiropyran-Merocyanine Reversion via Metal-Ion Selective Complexation: Trapping of the "Transient" cisMerocyanine Chem. Mater. 2001, 13, 2547-2551.

(33) Guo, X.; Zhang, D.; Zhang, G.; Zhu, D. Monomolecular Logic:"Half-Adder" based on Multistate/Multifunctional Photochromic Spiropyrans J. Phys. Chem. B 2004, 108, 11942-11945.

(34) Tanaka, M.; Nakamura, M.; Salhin, M. A. A.; Ikeda, T.; Kamada, K.; Ando, H.; Shibutani, Y.; Kimura, K. Synthesis and photochromism of spirobenzopyran derivatives bearing an oxymethylcrown ether moiety: Metal ion-induced switching between positive and negative photochromism J. Org. Chem. 2001, 66, 1533-1537.

(35) Tanaka, M.; Ikeda, T.; Xu, Q.; Ando, H.; Shibutani, Y.; Nakamura, M.; Sakamoto, H.; Yajima, S.; Kimura, K. Synthesis and Photochromism of Spirobenzopyrans and Spi- robenzothiapyran Derivatives Bearing Monoazathiacrown Ethers and Noncyclic Analogues in the Presence of Metal Ions J. Org. Chem. 2002, 67, 2223-2227.

(36) Inouye, M.; Noguchi, Y.; Isagawa, K. Sensitive and Selective Coloration of Cryptand-Type Crown Spirobenzopyrans for Alkaline-Earth Metal Cations Angew. Chem., Int. Ed. 1994, 33, 1163-1166.

(37) Piard, J. Influence of the Solvent on the Thermal Back Reaction of one Spiropyran J. Chem. Educ. 2014, 91, 21052111.

(38) Tian, W.; Tian, J. An Insight into the Solvent Effect on Photo-, Solvato-Chromism of Spiropyran through the Perspective of Intermolecular Interactions Dyes Pigm. 2014, 105, 66-74.

(39) Keum, S.; Roh, S.; Kim, S.; Lee, S.; Cho, C.; Kim, S.; Koh, K.-N. Unusual reverse photochromic behavior of indolinobenzospiropyran 6-carboxylates in aqueous binary solvents Bull. Korean Chem. Soc. 2006, 27, 187-188.

(40) Flannery Jr., J. B. Photo and Thermochromic Transients from substituted 1',3', $3^{\prime}-$ Trimethylindolinobenzospiropyrans J. Am. Chem. Soc. 1968, 90, 5660-5671.

(41) Roxburgh, C. J.; Sammes, P. G. On the Acid Catalysed Isomerisation of some Substituted Spirobenzopyrans Dyes Pigm. 1995, 27, 63-69.

(42) Schneider, S.; Grau, H.; Ringer, J. Surface-Enhanced Resonance Raman Studies of Spiropyrans (BIPS and derivatives) Mol. Cryst. Liq. Cryst. Sci. Technol., Sect. A 1994, 246, 267-274.

(43) Giordani, S.; Raymo, F. M. A Switch in a Cage with a Memory Org. Lett. 2003, 5, 3559-3562.

(44) Wu, Y.; Sasaki, T.; Kazushi, K.; Seo, T.; Sakurai, K. Interactions between Spiropyrans and Room-Temperature Ionic Liquids: Photochromism and Solvatochromism $J$. Phys. Chem. B 2008, 112, 7530-7536.

(45) Zhang, S.; Zhang, Q.; Ye, B.; Li, X.; Zhang, X.; Deng, Y. Photochromism of Spiropyran in Ionic Liquids: Enhanced Fluorescence and Delayed Thermal Reversion $J$. Phys. Chem. B 2009, 113, 6o12-6019.

(46) Raymo, F. M.; Giordani, S. Signal Processing at the Molecular Level J. Am. Chem. Soc. 20o1, 123, 4651-4652.

(47) Giordani, S.; Cejas, M. A.; Raymo, F. M. Photoinduced Proton Exchange between Molecular Switches Tetrahedron 2004, 6o, 10973-10981.

(48) Yamaguchi, T.; Maity, A.; Polshettiwar, V.; Ogawa, M. Negative Photochromism based on Molecular Diffusion between Hydrophilic and Hydrophobic Particles in the Solid State Inorg. Chem. 2018, 57, 3671-3674.

(49) Kinashi, K.; Nakamura, S.; Imamura, M.; Ishida, K.; Ueda, Y. The Mechanism for Negative Photochromism of Spiropyran in Silica J. Phys. Org. Chem. 2012, 25, 462-466.

(50) Kinashi, K.; Nakamura, S.; Ono, Y.; Ishida, K.; Ueda, Y. Reverse Photochromism of Spiropyran in Silica J. Photochem. Photobiol., A, 2010, 213, 136-140.

(51) Levy, D. Photochromic Sol-Gel Materials Chem. Mater. 1997, 9, 2666-2670.

(52) Evans, T. R.; Toth, A. F.; Leermakers, P. A. Medium effects of photochromism. Reversible Photobleaching of a Spiropyran on Silica Gel J. Am. Chem. Soc. 1967, 89, 506o5061.

(53) Zhang, F.; Zou, X.; Feng, W.; Zhao, X.; Jing, X.; Sun, F.; Rena, H.; Zhu, G. Microwave-Assisted Crystallization Inclusion of Spiropyran Molecules in Indium Trimesate Films with Antidromic Reversible Photochromism J. Mater. Chem. 2012, 22, 25019-25026.

(54) Piantek, M.; Schulze, G.; Koch, M.; Franke, K. J.; Leyssner, F.; Krüger, A.; Navío, C.; Miguel, J.; Bernien, M.; 
Wolf, M.; Kuch, W.; Tegeder, P.; Pascual, J. I. Reversing the Thermal Stability of a Molecular Switch on a Gold Surface: Ring-Opening Reaction of Nitrospiropyran $J$. Am. Chem. Soc. 2009, 131, 12729-12735.

(55) Qiu, Z.; Yu, H.; Li, J.; Wang, Y.; Zhang, Y. Spiropyranlinked Dipeptide Forms Supramolecular Hydrogel with Dual Responses to Light and to Ligand-Receptor Interaction Chem. Commun. 2009, 3342-3344.

(56) Wang, W.; Hu, J.; Zheng, M.; Zheng, L.; Wang, H.; Zhang, Y. Multi-Responsive Supramolecular Hydrogels based on Merocyanine-Peptide Conjugates Org. Biomol. Chem. 2015, 13, 11492-11498.

(57) Julià-López, A.; Hernando, J.; Ruiz-Molina, D.; GonzálezMonje, P.; Sedó, J.; Roscini, C. Temperature-Controlled Switchable Photochromism in Solid Materials Angew. Chem. Int. Ed. 2016, 55, 15044-15048.

(58) Burkinshaw, S. M.; Griffiths, J.; Towns, A. D. Reversibly Thermochromic Systems based on $\mathrm{pH}$-Sensitive Functional Dyes J. Mater. Chem. 1998, 8, 2677-2683.

(59) White, M. A.; LeBlanc, M. Thermochromism in Commercial Products J. Chem. Educ. 1999, 76, 1201-1205.

(6o) Vázquez-Mera, N.; Roscini, C.; Hernando, J.; RuizMolina, D. Liquid-Filled Capsules as Fast Responsive Photochromic Materials Adv. Optical Mater. 2013, 1, 631636.

(61) Vázquez-Mera, N.; Roscini, C.; Hernando, J.; RuizMolina, D. Liquid-Filled Valence Tautomeric Microcapsules: A Solid Material with Solution-Like Behavior Adv. Funct. Mater. 2015, 25, 4129 - 4134.

(62) Li, M.; Rouaud, O.; Poncelet, D. Microencapsulation by Solvent Evaporation: State of the Art for Process Engineering Approaches Int. J. Pharm. 2oo8, 363, 26-39.

(63) CRC Handbook of Chemistry and Physics, Internet Version, http://www.hbcpnetbase.com (accessed November 21, 2018); Lide, D. R. Ed.; CRC Press: Boca Raton, FL, 2005.

(64) The $T_{\mathrm{m}}$ of PDMS-OH is possibly below the detection limit of the DSC equipment $\left(-68^{\circ} \mathrm{C}\right)$.

(65) Kortüm, G.; Braun, W.; Herzog, G. Principles and Techniques of Diffuse-Reflectance Spectroscopy Angew. Chem. Int. Edit. 1963, 2, 333-341. 
SYNOPSIS TOC

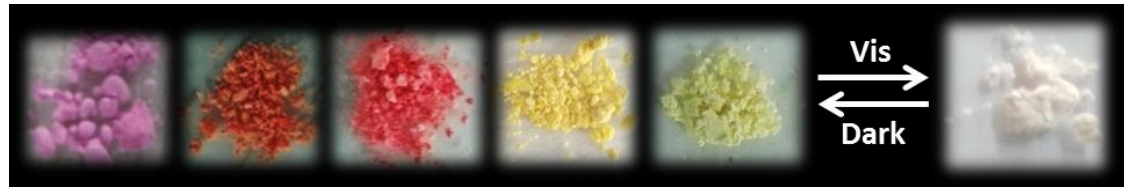

